\title{
Early Detection of Coronary Artery Flow and Myocardial Perfusion Impairment in Hypertensive Patients Evidenced by Myocardial Blush Grade (MBG) and Thrombolysis in Myocardial Infarction (TIMI) Frame Count (TFC)
}

\author{
Salvatore Evola, Francesco Cuttitta, Giovanna Evola, Francesca Macaione, Davide Piraino, \\ Maria Cristina Meschisi, Angelica Peritore, Daniela Di Lisi, \\ Guiseppina Novo and Salvatore Novo
}

\begin{abstract}
Objective In hypertensive patients with typical chest pain but absence of coronary stenosis the coronary microcirculation may be impaired, thus, our study aimed to appraise, in these subjects, the role of the coronary microcirculation, assessed by Myocardial Blush Grade (MBG) and Thrombolysis in Myocardial Infarction (TIMI) Frame Count (TFC).

Methods A total of 95 subjects with chest pain and uninjured coronary arteries were recruited into the study: 80 subjects were hypertensive while 15 subjects were normotensive. The hypertensive subjects were divided into two subgroups: hypertensive subjects with positive scintigraphy and hypertensive subjects with negative scintigraphy. The TFC, a quantitative method of assessing coronary artery flow, the MBG, an assessment of the level of tissue perfusion, and the Total Myocardial Blush Score (TMBS), the sum of the myocardial blush grades of each coronary territory, were evaluated on the coronary angiogram of every patient.

Results The TFC was higher $(\mathrm{p}<0.05)$ in hypertensive subjects than in normotensive subjects. The MBG and the TMBS were lower $(\mathrm{p}<0.05)$ in hypertensive subjects than in normotensive subjects. The TFC was higher $(\mathrm{p}<0.05)$ in positive scintigraphy than in negative scintigraphy. The MBG was lower $(\mathrm{p}<0.05)$ in positive scintigraphy than in negative scintigraphy. The Spearman rank correlation test showed that the TFC and the MBG was inversely associated.

Conclusion The hypertensive subjects had impaired coronary artery flow and myocardial perfusion, documented by the TFC, MBG and myocardial perfusion scintigraphy. These methods may be universally applicable, in fact they are validated and most catheterization laboratories have access to them.
\end{abstract}

Key words: hypertension, Myocardial Blush Grade, Thrombolysis in Myocardial Infarction Frame Count, angiography, angina

(Intern Med 51: 1653-1660, 2012)

(DOI: 10.2169/internalmedicine.51.7385)

\section{Introduction}

Hypertension is defined as systolic blood pressure $\geq 140$ $\mathrm{mmHg}$ and diastolic blood pressure $\geq 90 \mathrm{mmHg}$ (1). An important correlation has been shown between arterial hyper- tension and the clinical manifestations of ischemic heart disease $(2,3)$, and between the levels of pressure and number and entity of the atherosclerotic lesions (4). Because the earliest manifestation of cardiovascular disease are encountered in the microcirculation, the present study was aimed to appraise the mechanisms that produced myocardial ischemia in

Division and Post-Graduate School of Cardiology, Center for the Early Diagnosis of Preclinical and Multifocal Atherosclerosis and for the Secondary Prevention, University Hospital "P. Giaccone" of the University of Palermo, Italy

Received for publication January 23, 2012; Accepted for publication March 12, 2012

Correspondence to Dr. Francesca Macaione, francescamac85@libero.it 
Table 1. Baseline Characteristics of Patients

\begin{tabular}{|c|c|c|c|}
\hline & $\begin{array}{l}\text { Hypertensive Group } \\
(\mathrm{n}=\mathbf{8 0})\end{array}$ & $\begin{array}{l}\text { Normotensive Group } \\
(n=15)\end{array}$ & $\mathrm{p}$ value $\leq 0.05$ \\
\hline Females $n(\%)$ & $47(58.75 \%)$ & $7(46.66 \%)$ & 0.56 \\
\hline Males $n(\%)$ & $33(41.25 \%)$ & $8(53.33 \%)$ & 0.56 \\
\hline Mean Age $n \pm$ SD & $64 \pm 11$ & $69 \pm 9$ & 0.07 \\
\hline Smokers $n(\%)$ & $6(7.5 \%)$ & $2(13.33 \%)$ & 0.81 \\
\hline Family History of CVD $n(\%)$ & $27(33.75 \%)$ & $2(13.33 \%)$ & 0.20 \\
\hline Peripheral Atherosclerosis $n(\%)$ & $9(11.25 \%)$ & $0(0 \%)$ & 0.37 \\
\hline Central Obesity $n(\%)(M \geq 102, F \geq 88 \mathrm{~cm})$ & $22(27.5 \%)$ & $2(13.33 \%)$ & 0.40 \\
\hline$\uparrow$ Triglyceridemia $n(\%)(\geq 150 \mathrm{mg} / \mathrm{dL})$ & $7(8.75 \%)$ & $2(13.33 \%)$ & 0.94 \\
\hline$\uparrow \mathrm{TC} n(\%)(\geq 240 \mathrm{mg} / \mathrm{dL})$ & $36(45 \%)$ & $4(26.66 \%)$ & 0.30 \\
\hline$\uparrow$ Glycemia $n(\%)(\geq 126 \mathrm{mg} / \mathrm{dL})$ & $26(32.5 \%)$ & $4(26.66 \%)$ & 0.88 \\
\hline LVH & $46(57.5 \%)$ & $0(0 \%)$ & 0.0001 \\
\hline Aspirin $n(\%)$ & $29(36.25 \%)$ & $3(20 \%)$ & 0.36 \\
\hline Omeprazole $n(\%)$ & $25(31.25 \%)$ & $2(13.33 \%)$ & 0.27 \\
\hline Metformin $n(\%)$ & $16(20 \%)$ & $2(13.33 \%)$ & 0.80 \\
\hline Insulin $n(\%)$ & $5(6.20 \%)$ & $2(13.33 \%)$ & 0.66 \\
\hline B-Blockers $n(\%)$ & $22(27.5 \%)$ & $3(20 \%)$ & 0.78 \\
\hline $\operatorname{ACE} n(\%)$ & $30(37.5 \%)$ & $6(40 \%)$ & 0.91 \\
\hline Ca-Antagonists $n(\%)$ & $14(17.5 \%)$ & $2(13.33 \%)$ & 0.98 \\
\hline Nitrates $n(\%)$ & $6(7.5 \%)$ & $0(0 \%)$ & 0.60 \\
\hline Statins $n(\%)$ & $33(41.25 \%)$ & $4(26.66 \%)$ & 0.44 \\
\hline Clopidogrel $n(\%)$ & $18(22.5 \%)$ & $2(13.33 \%)$ & 0.64 \\
\hline Diuretics $n(\%)$ & $28(35 \%)$ & $3(20 \%)$ & 0.40 \\
\hline
\end{tabular}

hypertensive patients with typical chest pain and absence of coronary stenosis; particularly we tried to appraise the role of the coronary microcirculation valued by Myocardial Blush Grade (MBG) (5) and comparing the results so gotten with the assessment of the coronary flow obtained using the Thrombolysis in Myocardial Infarction (TIMI) Frame Count (TFC) $(6,7)$.

\section{Materials and Methods}

\section{Study population sample}

A total of 95 subjects were recruited among 1,200 subjects whose angiographic studies had been performed in our Department of Cardiovascular Disease between January 2010 and November 2010. Included were patients with typical chest pain, positive treadmill stress test, and normal coronary angiogram (absence of coronary stenosis). Excluded were patients with tortuous coronary arteries and coronary stenosis.

A total of 54 patients out of 80 enrolled hypertensive patients, as clinically indicated prior to angiographic study, had undergone myocardial perfusion scintigraphy upon entry in our department.

All patients enrolled underwent an accurate anamnesis, objective exam, echocardiography and haematochemical measurements, the database compiled for each patient contains the data listed in the Table 1.

A familial history of coronary artery disease (CAD) was defined as a coronary event occurring before 55 and 65 years, for first-degree males and females, respectively. Diabetes mellitus was defined as a fasting glucose $\geq 126 \mathrm{mg} / \mathrm{dL}$ on at least two separate occasions or as the use hypoglyce- mic drugs. Hypertension was defined as a blood pressure $>140 / 90 \mathrm{mmHg}$ or as use antihypertensive drugs. Patients were defined as smokers if they were current smokers or if they had stopped in less than 1 year. Body mass index was calculated as weight in kilograms divided by the square of height in meters. Waist circumference was measured at level of the right iliac crest. Central obesity was defined as a waist circumference $\geq 102 \mathrm{~cm}$ for males and $\geq 88 \mathrm{~cm}$ for females.

A blood sample was drawn in the morning, before the medical examination, after a 12-to-14 hour overnight fast. Total cholesterol and triglycerides were quantified by standard enzymatic-colorimetric method (8). Elevated levels of total cholesterol and triglycerides were defined as $\geq 240 \mathrm{mg}$ / $\mathrm{dL}$ and $\geq 150 \mathrm{mg} / \mathrm{dL}$, respectively.

\section{Exercise treadmill ECG testing}

Symptom-limited exercise treadmill-ECG testing with standard Bruce protocol was performed in all patients. A positive test was defined as $>0.1 \mathrm{mV}$ of flat or downsloping ST-segment depression $0.08 \mathrm{~s}$ from the $\mathrm{J}$ point.

\section{Coronary angiography}

All patients underwent coronary angiography. The coronary angiograms were analyzed by an experienced reader blinded to the results of treadmill-ECG. Each epicardial coronary vessel (left main, left anterior descending, circumflex, and right coronary artery) was assessed, and the visual estimation of the percent luminal reduction for each lesion was reported. Multiple projections were acquired to discern the maximal coronary artery luminal narrowing. Angiographic abnormalities were considered significant if $50 \%$ or greater luminal diameter stenosis was found in any epicar- 
dial coronary vessel.

\section{Thrombolysis in Myocardial Infarction Frame Count and Myocardial Blush Grade}

The TFC $(6,7)$ and the MBG (5) were evaluated on all coronary angiograms, which were filed on digital support. They were measured with a "Philips Integris 2000" by two independent observers blinded to the selection data. All angiograms were performed with 6 Fr guiding catheters.

The TFC, a quantitative method of assessing coronary artery flow, was evaluated on the three main coronary branches $(\mathrm{LAD}=$ left anterior descending coronary artery; LCX = left circumflex artery; RCA = right coronary artery), using the protocol described by Gibson et al. $(6,7)$. It was defined as the number of cineframes required for contrast to reach a standardized distal coronary landmark. These landmarks were as follows: the first branch of the posterolateral artery in the RCA, the distal branch of the lateral left ventricular wall artery furthest from the coronary ostium in the circumflex system, and the distal bifurcation known as the "moustache", "pitch fork" or "whale's tail" in the left anterior descending artery. By dividing the TFC of the LAD by a factor of 1.7 the correct-TFC (cTFC) was calculated. This correction factor of 1.7 is the average quotient between (mean normal population values) LAD frame count, 36.2 frames and RCA, 20.4 frames and LCX, 22.2 frames $(=36,2 /[(20,4+22,2) / 2]:$ it allows correction of raw measured values for flow differences due to the larger vessel length of the LAD.

The TFC was multiplied by a factor of correction to convert to a speed of 30 frame/second making direct comparison with earlier reports possible. In general, the TFC in the LAD and in the LCX were assessed in a right anterior oblique projection with caudal angulation (right anterior oblique caudal view) and TFC in the RCA was assessed in a left anterior oblique projection with cranial angulation (left anterior oblique cranial view).

The MBG, an assessment of the level of tissue perfusion, was defined in the following way: Grade 0, no or minimal blush; Grade 1, stain present and blush persistent on next injection; Grade 2, dye strongly persistent at end of washout; Grade 3, normal ground glass appearance of blush and dye mildly persistent at end of washout. It was evaluated on the three main coronary branches (LAD, LCX, RCA), using the protocol described by Gibson et al. (5). In general, the MBG in the LAD was assessed in a right anterior oblique projection with cranial angulation, in the LCX was assessed in a right anterior oblique projection with caudal angulation, and in the RCA was assessed in the left lateral view. Reproducibility of the visual assessment of the MBG and of corrected quantification of TFC was studied in a random sample of 25 patients. We found good reproducibility with intraobserver and interobserver variabilities of $87 \%$ and $92 \%$, respectively.

In addition we evaluated the total myocardial blush Score (TMBS) $(9,10)$ which was the sum of the myocardial blush grades of each coronary territory. We considered a TMBS value of 6-7 as "low" and a TMBS value of 8-9 as "normal".

\section{9mTc-sestamibi}

The clinical history of the patients revealed that myocardial perfusion SPECT was performed with a 2-d stressrest protocol. Each patient received $800-925 \mathrm{MBq}{ }^{99 m} \mathrm{Tc}-$ sestamibi intravenously either at baseline or during exercise stress testing. The segmental myocardial perfusion defects were matched to the arteries responsible for these perfusion defects. The septal and anterior myocardial segments were matched to the left anterior descending coronary artery; the inferior, inferoapical, and posterior myocardial segments were matched to the right coronary artery; and the lateral myocardial segments were matched to the left circumflex coronary artery.

\section{Echocardiography}

The clinical history of the patients revealed that M-mode, 2D and Doppler echocardiography were performed using a standard protocol. Two dimensional guided M- mode measurements were made according to the recommendations of the American Society of Echocardiography (ASE) (11). Left ventricular internal dimension, posterior wall thickness and interventricular septal thickness were measured at enddiastole and end-systole. Where optimal M-mode imaging could not be obtained, 2D linear measurements were obtained according to the ASE criteria (11). Left ventricular mass was calculated using the formula of Devereux and Reichek (12). The left ventricular mass was indexed to height 2 (H 2) and also to body surface area (BSA) to obtain the left ventricular mass index (LVMI). Left ventricular hypertrophy (increased LVMI) was defined as LVM/H2 $>77.7 \mathrm{~g} / \mathrm{m}^{2}$ in males and $>69.8 \mathrm{~g} / \mathrm{m}^{2}$ in females (12) and LVM/BSA $>134 \mathrm{~g} / \mathrm{m}^{2}$ in males and $>110 \mathrm{~g} / \mathrm{m}^{2}$ in females (13).

\section{Statistical analysis}

Data was analyzed using MedCalc software version 11.3.0.0. Continuous variables, as compared using Student's unpaired test, are presented as mean \pm standard deviation. Categorical variables, as compared with the "Chi-square" test when appropriate (expected frequency $>5$ ), are presented as counts and percentages. Otherwise, the Fisher's exact test was used.

Correlations were analyzed by Spearman rank correlation test.

$\mathrm{P}$ value equal or less than 0.05 was considered statistically significant.

\section{Results}

Two groups of patients were individualized in the study population according to the presence/absence of hypertension: hypertensive group and normotensive group. 
Table 2. Comparison TFC, MBG and TMBS between Hypertensive and Normotensive Subject

\begin{tabular}{llll}
\hline & Hypertensives $\mathbf{n}=\mathbf{8 0}$ & Normotensives $\mathbf{n = 1 5}$ & $\mathbf{p}$ value $\leq \mathbf{0 . 0 5}$ \\
\hline cTFC LAD & $25 \pm 7.7$ & $17.1 \pm 4.1$ & 0.0002 \\
TFC LCX & $25.9 \pm 6.5$ & $19.3 \pm 4.2$ & 0.0003 \\
TFC RCA & $18.6 \pm 6.7$ & $13.9 \pm 4.1$ & 0.0103 \\
MBG LAD & $2.5 \pm 0.5$ & $2.9 \pm 0.3$ & 0.0036 \\
MBG LCX & $2.4 \pm 0.4$ & $2.9 \pm 0.3$ & $<0.0001$ \\
MBG RCA & $2.3 \pm 0.4$ & $2.8 \pm 0.4$ & $<0.0001$ \\
TMBS & $7.3 \pm 1.2$ & $8.6 \pm 0.7$ & 0.0001 \\
\hline
\end{tabular}

TFC, Thrombolysis in Myocardial Infarction (TIMI) Frame Count; cTFC, corrected TFC; MBG, Myocardial Blush Grade; TMBS, Total Myocardial Blush Score; LAD, left anterior descending coronary artery; LCX, left circumflex artery; RCA, right coronary artery.

Table 3. Rank Correlation between MBG and TFC

\begin{tabular}{|c|c|c|c|}
\hline Variable $\mathbf{Y} / \mathbf{X}$ & $\begin{array}{l}\text { Spearman's coefficient of } \\
\text { rank correlation (rho) }\end{array}$ & $\begin{array}{c}\text { Confidence Intervals } \\
(95 \% \mathrm{CI})\end{array}$ & $p$ value $\leq 0.05$ \\
\hline $\begin{array}{l}\text { MBG LAD/ } \\
\text { cTFC LAD }\end{array}$ & -0.602 & -0.716 to -0.455 & $<0.0001$ \\
\hline $\begin{array}{l}\text { MBG LCX/ } \\
\text { TFC LCX }\end{array}$ & -0.472 & -0.615 to -0.299 & $<0.0001$ \\
\hline $\begin{array}{l}\text { MBG RCA/ } \\
\text { TFC RCA }\end{array}$ & -0.280 & -0.456 to -0.0837 & 0.0059 \\
\hline
\end{tabular}

Hypertensive group included a total of 80 patients, 33 males $(41.25 \%)$ and 47 females $(58.75 \%)$ with an average age of $64 \pm 11$ years (Table 1 ).

The normotensive group included a total of 15,8 males $(53.33 \%)$ and 7 females $(46.66 \%)$ with average age $68.94 \pm$ 11.31 years (Table 1 ).

The frequency of hypertension and left ventricular hypertrophy (LVH) [diagnosed by echocardiography] was higher in the hypertensive group than in normotensive group (n [\%] 46 [58\%] vs. 0 [0\%] p=0.0001). The frequency of other conventional risk factors was similar in the two groups. The patients' characteristics are indicated in Table 1.

The two groups (hypertensive and normotensive subjects) were homogeneous regarding medications, but the normotensive group could not be considered as well controlled hypertensive subjects because these subjects had no history of hypertension.

The TFC was significantly higher in the LAD, LCX and RCA of hypertensive subjects than in the respective coronary branches of normotensive subjects (Table 2).

The MBG and the TMBS were significantly lower in the LAD, LCX and RCA of hypertensive subjects than in the respective coronary branches of normotensive subjects (Table 3).

The Spearman rank correlation test showed that the TFC of LAD, LCX and RCA was inversely associated with MBG of the respective coronary branches, especially in the LAD $(\mathrm{r}=-0,602 ; \mathrm{p}<0.001)$ (Table 3).

Fifty-four hypertensive subjects underwent the myocardial perfusion scintigraphy and two subgroups were individualized in this group: hypertensive subjects with positive scintigraphy $(\mathrm{n}=37)$ and hypertensive subjects with negative scintigraphy $(\mathrm{n}=17)$.
The two groups, hypertensive subjects with positive scintigraphy and hypertensive subjects with negative scintigraphy, were homogeneous regarding left ventricular hypertrophy $(\mathrm{p}=0.2515)$, (data not shown).

In the hypertensive subjects with positive scintigraphy we evaluated the TFC and the MBG on artery responsible for the perfusion defect (IVA in 19 cases, LCX in 8 cases, RCA in 9 cases), while in hypertensive subjects with negative scintigraphy we evaluated the TFC and the MBG in the LAD, LCX and RCA, and we compared the respective values.

In all three cases the TFC of artery responsible for the perfusion defect was significantly higher (Table 4).

In all three cases the MBG of artery responsible for the perfusion defect was significantly lower (Table 4).

\section{Discussion}

The present our study shows that the TFC is a useful parameter for the assessment of coronary microvascular abnormalities and that the MBG (as well as the TMBS) could be a reliable marker of microvascular dysfunction well correlated to the TFC and myocardial scintigraphy. An interesting data, which was derived from this study, is that the area of positive scintigraphy is restricted in coronary artery territory in each group, we do not know the reason for this data but we can consider that as the initial phase of myocardial ischemia. Moreover we have detected that there is an important coincidence between the artery responsible for the perfusion defect and the artery with the longer TFC and the worst MBG in the patient with positive scintigraphy, this data offers a reflection for a better understanding of the pathophysiology of the microcirculation in hypertensive sub- 
Table 4. Comparison TFC and MBG between Positive Scintigraphy and Negative Scintigraphy

\begin{tabular}{|c|c|c|c|c|c|}
\hline & \multicolumn{2}{|c|}{$\begin{array}{l}\text { Negative Scintigraphy } \\
\mathrm{n}=17\end{array}$} & \multicolumn{2}{|c|}{$\begin{array}{l}\text { Positive Scintigraphy } \\
\mathbf{n}=\mathbf{3 7}\end{array}$} & \multirow{2}{*}{$p$ value $\leq 0.05$} \\
\hline & $\mathrm{n}$ & cTFC & $\mathrm{n}$ & cTFC & \\
\hline LAD & 17 & $25.2 \pm 10$ & 19 & $35.8 \pm 9$ & 0.0020 \\
\hline LCX & 17 & $24.7 \pm 7$ & 8 & $35.5 \pm 5$ & $<0.0001$ \\
\hline \multirow[t]{3}{*}{ RCA } & 17 & $17 \pm 5$ & 10 & $27.4 \pm 7$ & 0.0001 \\
\hline & \multicolumn{2}{|c|}{$\begin{array}{l}\text { Negative Scintigraphy } \\
\mathrm{n}=17\end{array}$} & \multicolumn{2}{|c|}{$\begin{array}{l}\text { Positive Scintigraphy } \\
\mathbf{n}=\mathbf{3 7}\end{array}$} & \multirow{2}{*}{$p$ value $\leq \mathbf{0 . 0 5}$} \\
\hline & $\mathrm{n}$ & MBG & $\mathrm{n}$ & MBG & \\
\hline LAD & 17 & $2.52 \pm 0.51$ & 19 & $1.8 \pm 0.5$ & 0.0001 \\
\hline LCX & 17 & $2.41 \pm 0.50$ & 8 & $1.8 \pm 0.2$ & 0.0031 \\
\hline RCA & 17 & $2.47 \pm 0.51$ & 10 & $1.74 \pm 0.4$ & 0.0007 \\
\hline
\end{tabular}

ject. The two groups (hypertensive and normotensive subjects) were significantly different regarding hypertension and LVH (diagnosed by echocardiography) but were homogeneous regarding other risk factors (Table 1) and medications. Consequently the difference in TFC, MBG and TMBS between hypertensive and normotensive subjects (Table 2) could be attributable to the hypertension and LVH. In addition, several studies, have shown that TFC and MBG are appropriate diagnostic tests to assess the coronary artery flow and the myocardial perfusion $(5-7,9)$, also in patients with normal coronary angiography. Our study showed that patients with hypertension had an important and early impairment of coronary blood flow and myocardial perfusion. In fact a transient increases in intravascular pressure significantly depresses endothelium-dependent vasodilatation in human arteries (14). Our study does not identify the precise mechanism by which increased intravascular pressure induces endothelial dysfunction but it suggests that elevated blood pressure per se may cause endothelial dysfunction in humans and may have implications for the pathophysiology of endothelial dysfunction in hypertension. In accordance with these results, many authors, using several modalities to investigate the relationship between hypertension and coronary microvascular circulation, showed that patients with hypertension had a coronary microvascular dysfunction. Geltman et al. (15) investigated myocardial perfusion using positron emission tomography in patients with chest pain and normal coronary arteries and they demonstrated that these patients had abnormalities of myocardial perfusion. Kawecka-Jaszcz et al. (16) investigated myocardial perfusion using cardiovascular magnetic resonance in patients with arterial hypertension and a history of chest pain and normal coronary angiography, and they demonstrated that these patients had regional myocardial perfusion reserve impairment which was independent of the presence of left ventricular hypertrophy. Additionally, many authors (17-19) demonstrated the presence of angina and coronary flow reserve diminished in hypertensive patients without left ventricular hypertrophy. Then, according to literature, the difference in TFC, MBG and TMBS between hypertensive and normotensive subjects (Table 2) showed that hypertension was responsible for impairment of coronary blood flow and myo- cardial perfusion, probably independent of the presence of left ventricular hypertrophy, and involved in determinism of angina. The TFC, a quantitative method of assessing coronary artery flow, was significantly higher in hypertensive subjects and in subjects with positive scintigraphy than in normotensive subjects and in subjects with negative scintigraphy. These abnormalities of TFC may be a reflection of structural or functional changes in the epicardial coronary arteries and/or alterations of coronary blood flow. Structural abnormalities in the epicardial coronary arteries of hypertensive subjects include hypertrophy of the vascular wall which result in decreased lumen size and increased wall: lumen ratio and consequently increased vascular resistance, but structural abnormalities include also development of invaginations and irregular profiles of the vascular wall. Several studies (20) have demonstrated that functional abnormalities of epicardial coronary arteries of hypertensive subjects include alteration in flow-mediated vasodilatation.

The MBG, an assessment of the level of tissue perfusion, was significantly lower in hypertensive subjects and in subjects with positive scintigraphy than in normotensive subjects and in subjects with negative scintigraphy. These alterations in hypertension may be a reflection of structural or functional changes in the microvessels $(21,22)$. Structural abnormalities in vessels of hypertensive patients include a decreased luminal diameter and reduction in density of vessels per volume of tissue (known as rarefaction) (23-25). There is much evidence that the development of hypertension is accompanied by rarefaction of arterioles and capillaries in both animal models and humans (26). Rarefaction can be either functional, when it is the result of vasoconstriction strong enough to close the vascular lumen and to result in the non-perfusion of a capillary bed, or structural, in which case the vessels are absent or with a decreased density in perfused tissues. It has been suggested that rarefaction may occur in two phases. The first phase "functional rarefaction" involves constriction of the microvessel to the point of nonperfusion. The non-perfused vessels may then degenerate, leading to the second phase "structural rarefaction". Microvessel rarefaction may be caused either by haemodynamic factors (such as changes in microvascular pressure and flow), or by the action or depletion of locally acting trophic 
or growth factors (such as fibroblast growth factor, transforming grown factor- $\beta$ or angiotensin II) (27).

The difference in TFC and MBG between hypertensive subjects with positive scintigraphy and hypertensive subjects with negative scintigraphy (Table 4) showed that the hypertensive subjects with positive scintigraphy had greater impairment of the coronary artery flow and the myocardial perfusion than those with negative scintigraphy and that myocardial perfusion scintigraphy might be a non invasive and an appropriate diagnostic test to identify early changes of the coronary microcirculation $(28,29)$. In fact, in accordance with the results obtained in the present study, perfusion abnormalities have been described in asymptomatic patients with systemic hypertension (30). The accuracy of myocardial perfusion scintigraphy in the detection of significant coronary artery disease has been reported to be in the range of $85-98 \%$ (31), but the issue of whether abnormal scan findings represent true perfusion changes or may be interpreted as false-positive remains controversial in nuclear cardiology. Arguments in favor of false-positive results are based on the presence of artefacts, whereas arguments for true coronary flow abnormalities are based on the presence of microcirculatory impairment or endothelial dysfunction. Although the evidence of a role for small-vessel disease is good, Wiedermann et al. (32) reported that in most patients presenting with this syndrome, intravascular sonography also shows abnormal epicardial coronary arteries. Early atheromatous plaques and intimal thickening, missed by coronary angiography, were associated with an abnormal (constrictive) response to exercise. This anatomic and physiologic heterogeneity in patients with angina and normal coronary angiography findings has been confirmed in a combined intracoronary sonography and Doppler flow velocity study by Erbel et al. (33). In that study, occult atherosclerosis and plaque formation were found in the left anterior descending and left main coronary arteries of $48 \%$ of 44 patients with chest pain and normal coronary angiography findings. Of the 44 patients included in that study, SPECT images were available for only $29(66 \%)$ but showed abnormal findings in $93 \%$ of patients with abnormal sonography findings and in only $28 \%$ of patients with normal vessels. Verna et al. (34) investigated the coronary arteries in 20 consecutive patients who had chest pain, normal coronary angiography findings, and positive stress-rest sestamibi SPECT findings, by intravascular sonograms and Doppler guidewire. They showed that early signs of atherosclerosis revealed by coronary sonography were more frequent, and usually more advanced, in the coronary territory corresponding to myocardial perfusion defects than in the reference territories. In addition, with the Doppler guidewire technique they evaluated regional coronary flow velocity reserve (CFR) in the bed distribution of either the abnormal scintigraphic segment or the reference regions. A reduction in coronary flow reserve was more frequent in the distribution of perfusion defects than in the reference territory. Aguirre et al. (35) showed, in patients with systemic hypertension and normal coronary an- giographies, that positive scintigraphy was a reflection of segmentary reduction of coronary reserve determined by intracoronary Doppler.

The present our study provided new evidence supporting the concept that an abnormal scintigraphy finding, rather than being false-positive, may be an early marker of vasomotion changes associated with occult atherosclerosis in patients with angina and normal coronary angiography findings, although vasomotor response to exercise cannot be assessed by MBG or TFC. Additionally, we demonstrated that these patients had segmental myocardial perfusion defects which was independent of the presence of left ventricular hypertrophy, because the two subgroups (hypertensive subjects with positive scintigraphy $[n=37]$ and hypertensive subjects with negative scintigraphy $[n=17])$ were homogeneous regarding left ventricular hypertrophy $(\mathrm{p}=0.2516)$.

In conclusion, it is very important that physicians identify individuals with coronary microvascular endothelial dysfunction (36), and in the present study we showed that the TFC, the MBG so also the TMBS, might be useful parameters to evaluate coronary microvascular dysfunction. In fact the Spearman rank correlation test showed that the TFC and the MBG were well correlated (Table 3). The TIMI and MBG are accurate and precise. Their simplicity and ease of use should facilitate broad application to provide further insight into pathophysiology: we anticipate that the TIMI will become a widely used method to evaluate coronary blood flow.

\section{Limitations}

Several limitations of this study should be considered. First, our study population was relatively small and the control group was small. We measured coronary flow by the TFC method. We did not use a Doppler coronary flow wire.

Additionally we did not use intravascular sonograms to study the vascular epicardial wall. But the TFC method is validated and robust, and we believe that the results of our study are clinically valid. Further most catheterization laboratories do not have access to a flow wire and intravascular sonograms, whereas the TFC method is universally applicable.

We used the myocardial blush grade classification described by Gibson and associates (5). A slightly different classification has been described by van't Hof et al. (37). It is possible that the relation between coronary flow pattern and myocardial blush would have been slightly different if the classification described by van't Hof and colleagues had been used. However, the classification by Gibson and associates has been adopted in other recent studies and it is validated and robust.

The two groups (hypertensive and normotensive subjects) were significantly different regarding left ventricular hypertrophy which may be involved in the alterations of TFC and MBG, but many authors (16-18) demonstrated the presence of angina and coronary flow reserve diminished in hypertensive patients without left ventricular hypertrophy. 
The present our study population had received extensive medical treatment before going to the catheterization laboratory, but the use of drugs of various classes did not differ between the study groups.

We did not study endothelial function of our study population therefore we could not demonstrate a positive correlation between endothelial dysfunction and alterations of TFC and MBG.

Lastly, we did not measure of the levels of marker of inflammation (C-reactive protein and/or Fibrinogen). As the majority of patients were transferred from another hospital, we did not have access to all of the C-reactive protein and/ or fibrinogen concentrations of these patients before the procedure, and the different hospitals that refer patients to us have different protocols and different assays for these markers, therefore we could not demonstrate a positive correlation between C-reactive protein and TIMI (thrombolysis in myocardial infarction) frame count in patients with slow coronary flow.

\section{Conclusion}

This study is a confirmation that the TFC and the MBG may be universally applicable, in fact these methods are validated and robust and most catheterization laboratories have access to these methods. In addition our findings suggest that the tendency to dismiss abnormal exercise perfusion findings as false-positive in patients with chest pain, positive exercise testing and normal coronary angiogram may be unjustified. Finally, this study is a confirmation that investigation of the microcirculation may serve to detect impending complications and provide an opportunity for early intervention.

The authors state that they have no Conflict of Interest (COI).

\section{References}

1. Guidelines for the management of arterial hypertension. The Task Force for the Management of Arterial Hypertension of the European Society of Hypertension (ESH) and of the European Society of Cardiology (ESC). Eur Heart J 28: 1462-1536, 2007.

2. Wilson PWF, Kannel WB. Hypertension, other risk factors, and the risk of cardiovascular disease. In: Hypertension: Pathophysiology, Diagnosis, and Management. 2nd edition. Laragh JH, Brenner BM, Eds. Raven Press, New York, 1995: 99-114.

3. Novo S. Ipertensione arteriosa e patologia cardiaca. Linee guida diagnostico-terapeutiche a cura della commissione congiunta ANMCO-SIC-SIIA. G. Ital Cardiol 29: 341-356, 1999 (in Italian).

4. Luisiani L, Visona A, Pagnan A. Noninvasive study of arterial hypertension and carotid atherosclerosis. Stroke 21: 410-414, 1990.

5. Gibson CM, Cannon CP, Murphy SA, et al. For the TIMI 10B investigators. The relationship of the TIMI myocardial perfusion grade to mortality after administration of thrombolytic drugs. Circulation 101: 125-130, 2000.

6. Gibson CM, Cannon CP, Daley WL, et al. For the TIMI 4 Study Group. TIMI frame count: a quantitative method for assessing coronary artery flow. Circulation 93: 879-888, 1996.

7. Gibson CM, Murphy SA, Rizzo MJ, et al. Relationship between
TIMI frame count and clinical outcomes after thrombolytic administration. Circulation 99: 1945-1950, 1999.

8. Murata M, Ide T. Determination of cholesterol in sub-nanomolar quantities in biological fluids by high-performance liquid chromatography. J Chromatogr 579: 329-333, 1992.

9. Atmaca Y, Duzen V, Ozdol C, et al. Total blush score: a new index for the assessment of microvascular perfusion in idiopathic dilated cardiomyopathy. Coron Artery Dis 19: 181-185, 2008.

10. Atmaca Y, Ozdemir AO, Ozdol C, et al. Angiographic evaluation of myocardial perfusion in patients with syndrome X. Am J Cardiol 96: 803-805, 2005.

11. Sahn DJ, DeMaria A, Kisslo J, Weyman A. Recommendations regarding quantitation in M-mode echocardiography: results of a survey of echocardiographic measurements. Circulation 58: 10721083, 1978.

12. Devereux RB, Reichek N. Echocardiographic determination of left ventricular mass in man. Anatomic validation of the method. Circulation 55: 613-618, 1977.

13. Schiller NB, Shah PM, Crawford M, et al. Recommendations for quantitation of the left ventricle by two-dimensional echocardiography. American Society of Echocardiography Committee on Standards, Subcommittee on Quantitation of Two-Dimensional Echocardiograms. J Am Soc Echocardiogr 2: 358-367, 1989.

14. Paniagua OA, Bryant MB, Panza JA. Transient hypertension directly impairs endothelium-dependent vasodilation of the human microvasculature. Hypertension 36: 941-944, 2000.

15. Geltman EM, Henes CG, Senneff MJ, Sobel BE, Bergmann SR. Increased myocardial perfusion at rest and diminished perfusion reserve in patients with angina and angiographically normal coronary arteries. J Am Coll Cardiol 16: 586-595, 1990.

16. Kawecka-Jaszcz K, Czarnecka D, Olszanecka A, et al. Myocardial perfusion in hypertensive patients with normal coronary angiograms. J Hypertens 26: 1686-1694, 2008.

17. Brush JE Jr, Cannon RO III, Schenke WH, et al. Angina due to coronary microvascular disease in hypertensive patients without left ventricular hypertrophy. N Engl J Med 319: 1302-1307, 1988.

18. Novo S, Barbagallo M, Abrignani MG, et al. Increased prevalence of cardiac arrhythmias and transient episodes of myocardial ischemia in hypertensives with left ventricular hypertrophy but without clinical history of coronary heart disease. Am J Hypertension 10: 843-851, 1997.

19. Novo S, Longo B, Liquori M, et al. Cardiac arrhythmias and transient episodes of myocardial ischemia in hypertensives with or without left ventricular hypertrophy. J Hypertens 10 (Suppl. 4): S21/P32, 1992.

20. Antony I, Lerebours G, Nitenberg A. Angiotensin-converting enzyme inhibition restores flow-dependent and cold pressor testinduced dilations in coronary arteries of hypertensive patients. Circulation 94: 3115-3122, 1996.

21. Shore AC, Tooke JE. Microvascular function in human essential hypertension. J Hypertens 12: 717-728, 1994.

22. Xiaoying L. Characteristics of coronary microvascular lesions in autopsied elderly with hypertensive left ventricular hypertrophy. Chin Med J 115: 658-663, 2002.

23. Folkow B. Structural factor in primary and secondary hypertension. Hypertension 16: 89-101, 1990.

24. Landau J, Davis E. Capillary thinning and high capillary blood pressure in hypertension. Lancet 269: 1327-1330, 1957.

25. Williams SA, Tooke JE, MacGregor G. Rarefaction of skin capillaries in hypertension. Clin Sci 70 (suppl 13): 14P, 1986.

26. Henrich HA, Romen W, Heimgärtner W, Hartung E, Bäumer F. Capillary rarefaction characteristic of the skeletal muscle of hypertensive patients. Klin Wochenschr 66: 54-60, 1988.

27. Prewitt RL, Hashimoto H, Stacy DL. Structural and functional rarefaction of microvessels in hypertension. In: Blood Vessel Changes in Hypertension: Structure and Function. Lee R, Ed. 
CRC Press, Boca Ranton, FL, 1990: 71-90.

28. Williams SA, Tooke JE. Noninvasive estimation of increased structurally-based resistance to blood flow in skin of subjects with essential hypertension. Int J Microcirc Clin Exp 11: 109-116, 1992.

29. Žižek B, Poredoš P. Endothelial dysfunction in hypertensive patients and in normotensive offspring of subjects with essential hypertension. Heart 85: 215-217, 2001.

30. Elhendy A. Impact of hypertension on the accuracy of exercise stress scintigraphy for the diagnosis of coronary artery disease. Heart 85: 655-661, 2001.

31. Houghton JL, Frank MJ, Carr AA, von Dohlen TW, Prisant LM. Relations among impaired coronary flow reserve, left ventricular hypertrophy and thallium perfusion defects in hypertensive patients without obstructive coronary artery disease. J Am Coll Cardiol 15: 43-51, 1990.

32. Wiedermann JG, Schwartz A, Apfelbaum M. Anatomic and physiologic heterogeneity in patients with syndrome $\mathrm{X}$ : an intravascular ultrasound study. J Am Coll Cardiol 25: 1310-1317, 1995.

33. Erbel R, Ge J, Bockisch A, et al. Value of intracoronary ultra- sound and Doppler in the differentiation of angiographically normal coronary arteries: a prospective study in patients with angina pectoris. Eur Heart J 17: 880-889, 1996.

34. Verna E, Ceriani L, Giovanella L, Binaghi G, Garancini S. "Falsepositive" myocardial perfusion scintigraphy findings in patients with angiographically normal coronary arteries: insights from intravascular sonography studies. J Nucl Med 41: 1935-1940, 2000.

35. Aguirre JM, Rodriguez E, Ruiz de Azua E, et al. Segmentary coronary reserve in hypertensive patients with $\mathrm{LVH}$, gammagraphic ischemia and normal coronary angiography. Eur Heart $\mathrm{J}$ 14: 25-31, 1993.

36. L'Abbate A, Sambuceti G. How to study coronary microvascular dysfunction and its clinical relevance. Ital Heart J 3: 230-236, 2002.

37. van't Hof AW, Liem A, Suryapranata H, Hoorntje JC, de Boer MJ, Zijlstra F. Angiographic assessment of myocardial reperfusion in patients treated with primary angioplasty for acute myocardial infarction: myocardial blush grade. Zwolle Myocardial Infarction Study Group. Circulation 9: 2302-2306, 1998.

(C) 2012 The Japanese Society of Internal Medicine http://www.naika.or.jp/imindex.html 